\title{
Biomedical insights into cell adhesion and migration-from a viewpoint of central nervous system tumor immunology
}

\author{
Mitsugu Fujita $^{1 *}$, Takaaki Matsui ${ }^{2}$ and Akihiko Ito $^{3}$ \\ ${ }^{1}$ Department of Microbiology, Faculty of Medicine, Kindai University, Osaka, Japan, ${ }^{2}$ Gene Regulation Research, Graduate \\ School of Biological Sciences, Nara Institute of Science and Technology, Nara, Japan, ${ }^{3}$ Department of Pathology, Faculty of \\ Medicine, Kindai University, Osaka, Japan
}

Keywords: cell adhesion, cell migration, cancer immunology, central nervous system, integrins, chemokines

\section{Fine-tuned Orchestration of Cell Adhesion and Migration}

\section{OPEN ACCESS}

Edited by:

Gerard Apodaca,

University of Pittsburgh, USA

Reviewed by:

Lorraine Santy,

The Pennsylvania State University,

USA

James Alan Marrs,

Indiana University-Purdue University

Indianapolis, USA

*Correspondence:

Mitsugu Fujita

mfujita47@gmail.com

Specialty section:

This article was submitted to

Cell Adhesion and Migration,

a section of the journal

Frontiers in Cell and Developmental

Biology

Received: 22 July 2015 Accepted: 08 September 2015

Published: 14 October 2015

Citation:

Fujita M, Matsui T and Ito A (2015) Biomedical insights into cell adhesion and migration-from a viewpoint of central nervous system tumor immunology.

Front. Cell Dev. Biol. 3:55. doi: 10.3389/fcell.2015.00055
Cell adhesion and migration is a central process in the development and maintenance of multicellular organisms (Friedl et al., 2012). Tissue formation during embryonic development, wound healing, and immune responses all require the fine-tuned, orchestrated movement of cells. In this regard, recent cutting-edge studies have elucidated how cell adhesion and migration achieve three-dimensional collective cell movement (Rørth, 2012; Matsui et al., 2015). That is, collective cell movement uses a mechanical guidance system where each cell in the cell groups individually but coordinately guides the global motion of the cell groups. This mechanism is involved in a variety of biological reactions such as embryonic morphogenesis, organogenesis, immune reactions, and/or carcinogenesis (Friedl and Gilmour, 2009). In this review article, we will particularly pick up immune reactions in central nervous system (CNS) and CNS tumors to look into the fine-tuned mechanisms how cell adhesion and migration are involved in physiological condition as well as pathological conditions.

\section{Significance of Cell Adhesion in CNS/CNS Tumor Immunology}

In the past, the CNS has been characterized as an immunologically privileged site. Nowadays, however, immune reactions are known to occur in the CNS (Ousman and Kubes, 2012). In particular, regarding immune cell trafficking to the brain, the mechanisms how leukocytes translocate from high velocity circulation into the brain parenchyma is explained as follows: (1) tethering/rolling, (2) activation, (3) adhesion, and (4) transmigration (Engelhardt, 2008). Briefly, leukocytes slow down on endothelial cells by selectin-mediated interactions expressed on both of the cell types. Then, at a reduced velocity, the leukocytes sense chemokines on the endothelial cells and become activated through G-protein signaling to upregulate integrins (Fujita et al., 2009). Finally, with this tight interaction in place, leukocytes transmigrate into the parenchyma to function. In the following section, we discuss major adhesion molecules that are relevant to this process.

\section{ICAM1-LFA1 Interaction for T Lymphocytes}

ICAM1 (Intercellular Adhesion Molecule 1), which is also known as CD54, is a cell surface glycoprotein that is typically expressed on endothelial cells as well as distinct subsets of leukocytes (Rothlein et al., 1986). ICAM1 is a ligand for LFA1 (Lymphocyte Function-Associated molecule 1), 
a receptor primarily expressed on leukocytes. As mentioned above, when leukocytes slow down via interactions between LFA1 on leukocytes and ICAM1 on endothelial cells, the leukocytes become activated by chemokine signals (we will discuss later) and further upregulate the expression levels of the integrins. In particular, the interaction between ICAM1 and LFA1 is a crucial step in the generation of tumor-specific cytotoxic T lymphocytes (CTLs) (Jenkinson et al., 2005). It is also required for the in situ activation and the migration of the CTLs (Bachmann et al., 1997). Moreover, ICAM1 has been shown to play a pivotal role in physical and functional interaction between CNS tumors and CTLs (Ueda et al., 2009). Therefore, malignant CNS tumor cells reduce the expression levels of ICAM1 to achieve their tumor immune escape (Dunn et al., 2007).

\section{VCAM1-VLA4 Interaction for T Lymphocytes}

VCAM1 (Vascular Cell Adhesion Molecule 1), which is also known as CD106, mediates the adhesion of lymphocytes, monocytes, eosinophils, and basophils to vascular endothelium (Cybulsky et al., 1991). It also functions in leukocyte-endothelial cell signal transduction. VCAM1 interacts with VLA4 (Very Late Antigen-4 or integrin $\alpha 4 \beta 1$ ) (Lin and Castro, 1998).

Expression of tissue-specific homing molecules directs antigen-experienced $\mathrm{T}$ lymphocytes to particular peripheral tissues. It is therefore essential to gain understanding of pivotal homing receptors that dictate CNS tumor-homing of $\mathrm{T}$ lymphocytes. It has been shown that in vivo imprinting of distinct homing phenotypes of $\mathrm{T}$ lymphocytes occurs in response to tumor-expressing antigens in intracerebral, subcutaneous, and intraperitoneal sites (Calzascia et al., 2005). In addition, CNShoming type-1 CTLs (Tc1; the most potent effector T cells) but not its counterpart type-2 CTLs (Tc2) preferentially express VLA4 (Sasaki et al., 2007; Zhu et al., 2007). As mentioned above, ICAM1-LFA1 interaction enhances a stable interaction between VCAM1 and VLA4, which allows the cells to migrate into brain parenchyma (Sasaki et al., 2008).

\section{CADM1-mediated Interaction for Dendritic Cells (DCs)}

CADM1 (Cell Adhesion Molecule 1) is an immunoglobulin superfamily member that is expressed on neuron cells such as superior cervical ganglions (Watabe et al., 2003). In addition, a soluble form of CADM1 (sCADM1) can be generated as an alternative splicing variant, which is involved in directional neuron extension (Hagiyama et al., 2009). CADM1 has been shown to interact with an intracytoplasmic protein DAL1 (Yageta et al., 2002).

DCs are known to be antigen-presenting cells (APCs) of the mammalian immune system; they act as messengers between the innate and the adaptive immune systems (Palucka and Banchereau, 2012). Their main function is to process antigen material and present it on the cell surface to naive Tlymphocytes to activate them. Therefore, they decide how $\mathrm{T}$ lymphocytes differentiate. Recent studies have shown that a certain subset of DCs express CADM1 (Dutertre et al., 2014). In addition, CADM1-postive and CADM1-negative subsets correspond to type-1 DCs (which induces type-1 $\mathrm{T}$ lymphocytes such as
Tc1) and type-2 DCs, respectively. These data suggest that CNS tumors may preferentially induce immunosuppressive milieu by promoting CADM1-negative DCs in the tumor microenvironment.

\section{Significance of Cell Migration in CNS/CNS Tumor Immunology}

In turn, regarding the cell migration in CNS immunology, the most important factors are chemokines because they act as a chemoattractant to guide the migration of leukocytes directly (Proudfoot, 2002). Chemokines are a family of cytokines and classified into four main subfamilies: CXC, CC, CX3C, and XC (Zlotnik and Yoshie, 2012). All of these proteins exert their biological effects by interacting with $G$ protein-linked transmembrane receptors called chemokine receptors that are selectively found on the surfaces of their target cells. Some chemokines are involved in immune surveillance; they direct lymphocytes to the lymph nodes so that the lymphocytes can screen for invasion of pathogens by interacting with APCs residing in these tissues. Some chemokines have roles in development; they promote angiogenesis or guide cells to tissues that provide specific signals critical for cellular maturation. Other chemokines are inflammatory; they are released from a wide variety of cells in response to bacterial infection. Inflammatory chemokines function mainly as chemoattractants for leukocytes to recruit them from the blood to sites of infection or tissue damage. In the following sections, we discuss about the CNS tumor-relevant immunology from a viewpoint of chemokines.

\section{CCR7-CCL19/CCL21 Axis for DCs}

CCR7 is a chemokine receptor that is expressed by various subsets of leukocytes, and its ligands are CCL19 and CCL21 (Förster et al., 2008). These chemokines are constitutively expressed and control cell movement during homeostasis. CCR7CCL19/21 chemokine axis is essentially involved in homing of activated DCs to the lymph nodes. Within lymph nodes of the systemic immune system, T lymphocytes establish close physical contacts with DCs, which allows their antigen-specific activation (Ganguly et al., 2013).

In contrast, in the CNS, a variety of cell populations have been postulated as primary APCs: vascular endothelial cells, smooth muscle cells, astrocytes, perivascular macrophages, choroid plexus epithelial cells, neurons, and DCs (Dunn et al., 2007). Presentation of CNS antigens by APCs can occur through multiple mechanisms (Walker et al., 2003): (1) APC uptake antigen within the CNS and migrate to lymph nodes to present antigens; (2) antigen drains to lymph nodes where APCs take them up to present; and (3) cells that express the antigen directly drain to lymph nodes and present their own antigen (direct presentation as opposed to cross presentation by DCs). In the process of lymph node-homing of DCs, CCR7 appears to play a central role in this process. Indeed, CCR7+ DCs injected in brain tumors have been shown to migrate to the cervical lymph nodes (CLNs) (Dunn et al., 2007; Fujita et al., 2009). Likewise, CCR7+ $\mathrm{CD} 11 \mathrm{c}+$ cells resembling classical bone marrow-derived DCs 
appear to be involved in other diseases such as CNS infectious and autoimmune encephalitis (Ganguly et al., 2013).

\section{CXCR3-CXCL10 Axis for T Lymphocytes}

CXCR3 is a chemokine receptor that is rapidly induced on naïve $\mathrm{T}$ lymphocyte following activation and preferentially remains highly expressed on type-1 helper (Th1)-type CD4+ $\mathrm{T}$ lymphocytes, effector CD8+ T lymphocytes and innate-type lymphocytes such as natural killer (NK) and NKT cells (Groom and Luster, 2011). CXCR3 is activated by three interferon (IFN)$\gamma$-inducible ligands CXCL9, CXCL10, and CXCL11. Among these chemokines, CXCL10 is induced by a variety of innate stimuli that induce IFN- $\alpha / \beta$ as well as the adaptive immune cell cytokine IFN- $\gamma$. CXCL10 has been attributed to several roles, such as (1) chemoattraction for monocytes/macrophages, $\mathrm{T}$ lymphocytes, NK cells, and dendritic cells; (2) promotion of T cell adhesion to endothelial cells; and (3) inhibition of bone marrow colony formation and angiogenesis.

In particular, Tc1 are known to efficiently traffic to the CNS tumor site through CXCR3-CCL10 axis compared with Tc2 (Nishimura et al., 2006; Zhu et al., 2007; Fujita et al., 2009). CXCR3 is uniquely up-regulated on Tc1, which is critical for efficient CNS tumor-homing of Tc1. Further information on mechanisms underlying efficient CNS-tumor homing of CTLs should be gained for development of truly effective immunotherapy strategies for CNS tumors.

\section{CCR2-CCL2 Axis for Macrophages/Microglia}

CCR2 is a chemokine receptor, which regulates the mobilization of monocytes from bone marrow to the inflammatory sites, and it has been extensively studied in CNS inflammation (Chu et al., 2014). CCR2 is activated by several chemokines, including CCL2, CCL7, CCL8, CCL12, CCL13, and CCL16. Activation of CCR2 results in directional migration of receptor-bearing cell types. Among them, CCL2 is known to be the most potent activator of CCR2 signaling, leading to monocyte transmigration. In the CNS tumor setting, CCL2 is secreted by tumor cells (Zhu et al., 2011). CCL2 can directly promote angiogenesis through the recruitment of tumor-associated macrophages (Salcedo et al., 2000). In addition, CCL2 has been shown to be critical for cell proliferation of CNS tumors, cancer cell metastasis, as well as tumor aggressiveness (Huang et al., 2007).

In the CNS, macrophages/microglial cells constitute the first line of cellular defense against a variety of stressors, participating in the regulation of innate and adaptive immune responses (Badie and Schartner, 2000). Many CNS tumors exhibit a prominent macrophage/microglia infiltrate. It is postulated that defense functions of macrophage/microglia against glioma are compromised in the tumor microenvironment. CNS macrophages/microglial cells expressed substantial levels of CCR2 and Toll-like receptors (TLRs), which are critical components for APCs to mediate innate immune responses to any infectious or traumatic challenge and activating adaptive immune responses. However, CNS macrophages/microglial cells do not appear to produce pro-inflammatory cytokines such as TNF- $\alpha$, IL-1, or IL-6. Moreover, these cells, in addition to decreased surface expression of MHC class II (Watters et al., 2005), lack expression of the costimulatory molecules CD86, CD80, and CD40 critical for $\mathrm{T}$ cell activation, thereby unable to activate $\mathrm{T}$ cells properly ex vivo (Hussain et al., 2006). Therefore, in the CNS tumor microenvironment, macrophages/microglial cells are considered to be immunosuppressive so that they are potent therapeutic target for anti-CNS tumor immunotherapy (Fujita et al., 2010, 2011; Zhu et al., 2011).

\section{CCR4-CCL22 Axis for Regulatory T Lymphocytes (Tregs)}

CD4+CD25+FoxP3+ Tregs are also found in the tumor microenvironment (Zou, 2006). There are four potential sources for Tregs in the CNS tumor microenvironment: the thymus, lymph nodes, bone marrow, and peripheral blood traffic to the tumor. Tregs express CCR4; abundant expression of CCL22, the ligand for CCR4, in the tumor microenvironment stimulates the tumor infiltration of Tregs. The tumor microenvironment contains molecules that can suppress APC differentiation and function. These dysfunctional APCs can in turn stimulate Treg differentiation. In addition, DCs can stimulate Treg expansion, and it is predicted that DCs in the tumor microenvironment move toward draining lymph nodes and further induce Treg expansion.

Similarly, the suppressive activity of Tregs is an important factor since they limit CTL-mediated destruction of CNS tumor cells. An increased ratio of CCR4+FoxP3+ Tregs to total $\mathrm{CD} 4+\mathrm{T}$ cells correlates with impairment of CD4+ $\mathrm{T}$ cell proliferation in peripheral blood specimens obtained from CNS tumor patients (Fecci et al., 2006; Hussain et al., 2006). Moreover, Tregs are not present in normal brain tissue but are very rarely found in low-grade gliomas and oligodendrogliomas (Fujita et al., 2008; Heimberger et al., 2008). These studies also observed that Tregs infiltration differs significantly in the tumors according to lineage, pathology, and grade. In addition, treatment of CNS tumor-bearing hosts with anti-CD25 mAb delayed the tumor growth and prolonged the survival, suggesting that CCR4+CD4+CD25+ Tregs play an important role in suppressing the immune response to CNS tumors (El Andaloussi et al., 2006).

\section{Perspectives}

We reviewed recent progress in the field of the CNS and CNS tumor immunology from a viewpoint of cell adhesion (primarily integrins) and migration (primarily chemokines). As discussed above, a line of studies have uncovered important roles of cell adhesion and migration during development of multicellular organisms as well as pathological conditions such as cancers. In addition, kinetics of cell adhesion and migration in the CNS provides a diverse scope for therapeutic strategies and target molecules. Moreover, it is clear that the CNS and CNS tumors are equipped with numerous layers of immunosuppression and immune escape mechanisms, perhaps including ones that we have not yet identified. These discoveries would allow us to develop strategies to overcome each of these mechanisms. The eventual success of tumor immunotherapies including those for CNS tumors will be dependent upon not only implementation of molecularly targeted trials that address multiple layers of 
challenges but also in-depth understanding of organ/tumorspecific immunology mediated by organ-specific cell adhesion and migration.

\section{Acknowledgments}

We thank Ms. Heather A. McDonald, BS, CCRC of Three Rivers Research Partners, LLC. for critical reading of the

\section{References}

Bachmann, M. F., McKall-Faienza, K., Schmits, R., Bouchard, D., Beach, J., Speiser, D. E., et al. (1997). Distinct roles for LFA-1 and CD28 during activation of naive $\mathrm{T}$ cells: adhesion versus costimulation. Immunity 7, 549-557. doi: 10.1016/S1074-7613(00)80376-3

Badie, B., and Schartner, J. M. (2000). Flow cytometric characterization of tumorassociated macrophages in experimental gliomas. Neurosurgery 46, 957-961. doi: 10.1097/00006123-200004000-00035

Calzascia, T., Masson, F., Di Berardino-Besson, W., Contassot, E., Wilmotte, R., Aurrand-Lions, M., et al. (2005). Homing phenotypes of tumor-specific CD8 T cells are predetermined at the tumor site by crosspresenting APCs. Immunity 22, 175-184. doi: 10.1016/j.immuni.2004.12.008

Chu, H. X., Arumugam, T. V., Gelderblom, M., Magnus, T., Drummond, G. R., and Sobey, C. G. (2014). Role of CCR2 in inflammatory conditions of the central nervous system. J. Cereb. Blood Flow Metab. 34, 1425-1429. doi: 10.1038/jcbfm.2014.120

Cybulsky, M. I., Fries, J. W., Williams, A. J., Sultan, P., Eddy, R., Byers, M., et al. (1991). Gene structure, chromosomal location, and basis for alternative mRNA splicing of the human VCAM1 gene. Proc. Natl. Acad. Sci. U.S.A. 88, 7859-7863. doi: 10.1073/pnas.88.17.7859

Dunn, G. P., Dunn, I. F., and Curry, W. T. (2007). Focus on TILs: Prognostic significance of tumor infiltrating lymphocytes in human glioma. Cancer Immun. 7, 12 .

Dutertre, C. A., Wang, L. F., and Ginhoux, F. (2014). Aligning bona fide dendritic cell populations across species. Cell Immunol. 291, 3-10. doi: 10.1016/j.cellimm.2014.08.006

El Andaloussi, A., Han, Y., and Lesniak, M. S. (2006). Prolongation of survival following depletion of $\mathrm{CD} 4+\mathrm{CD} 25+$ regulatory $\mathrm{T}$ cells in mice with experimental brain tumors. J. Neurosurg. 105, 430-437. doi: 10.3171/jns.2006.105.3.430

Engelhardt, B. (2008). The blood-central nervous system barriers actively control immune cell entry into the central nervous system. Curr. Pharm. Des. 14, 1555-1565. doi: 10.2174/138161208784705432

Fecci, P. E., Mitchell, D. A., Whitesides, J. F., Xie, W., Friedman, A. H., Archer, G. E., et al. (2006). Increased regulatory T-cell fraction amidst a diminished CD4 compartment explains cellular immune defects in patients with malignant glioma. Cancer Res. 66, 3294-3302. doi: 10.1158/0008-5472.CAN-05-3773

Förster, R., Davalos-Misslitz, A. C., and Rot, A. (2008). CCR7 and its ligands: balancing immunity and tolerance. Nat. Rev. Immunol. 8, 362-371. doi: $10.1038 /$ nri2297

Friedl, P., and Gilmour, D. (2009). Collective cell migration in morphogenesis, regeneration and cancer. Nat. Rev. Mol. Cell. Biol. 10, 445-457. doi: $10.1038 / \mathrm{nrm} 2720$

Friedl, P., Sahai, E., Weiss, S., and Yamada, K. M. (2012). New dimensions in cell migration. Nat. Rev. Mol. Cell. Biol. 13, 743-747. doi: 10.1038/nrm3459

Fujita, M., Kohanbash, G., Fellows-Mayle, W., Hamilton, R. L., Komohara, Y., Decker, S. A., et al. (2011). COX-2 blockade suppresses gliomagenesis by inhibiting myeloid-derived suppressor cells. Cancer Res. 71, 2664-2674. doi: 10.1158/0008-5472.CAN-10-3055

Fujita, M., Scheurer, M. E., Decker, S. A., McDonald, H. A., Kohanbash, G., Kastenhuber, E. R., et al. (2010). Role of type 1 IFNs in antiglioma immunosurveillance-using mouse studies to guide examination of novel prognostic markers in humans. Clin. Cancer Res. 16, 3409-3419. doi: 10.1158/1078-0432.CCR-10-0644 manuscript. We are grateful to the following grant supports: Ministry of Education, Culture, Sport, Science and Technology (MEXT), Ministry of Health, Labor and Welfare (MHLW), Walter L. Copeland Foundation, Aichi Cancer Research Foundation, Kinki University On-Campus Research Grant, Nakajima Foundation, Mochida Memorial Foundation, Sagawa Foundation, Takeda Science Foundation, and Uehara Memorial Foundation.

Fujita, M., Zhu, X., Sasaki, K., Ueda, R., Low, K. L., Pollack, I. F., et al. (2008). Inhibition of STAT3 promotes the efficacy of adoptive transfer therapy using type-1 CTLs by modulation of the immunological microenvironment in a murine intracranial glioma. J. Immunol. 180, 2089-2098. doi: 10.4049/jimmunol.180.4.2089

Fujita, M., Zhu, X., Ueda, R., Sasaki, K., Kohanbash, G., Kastenhuber, E. R., et al. (2009). Effective immunotherapy against murine gliomas using type 1 polarizing dendritic cells-significant roles of CXCL10. Cancer Res. 69, 1587-1595. doi: 10.1158/0008-5472.CAN-08-2915

Ganguly, D., Haak, S., Sisirak, V., and Reizis, B. (2013). The role of dendritic cells in autoimmunity. Nat. Rev. Immunol. 13, 566-577. doi: 10.1038/ nri3477

Groom, J. R., and Luster, A. D. (2011). CXCR3 ligands: redundant, collaborative and antagonistic functions. Immunol. Cell Biol. 89, 207-215. doi: 10.1038/icb.2010.158

Hagiyama, M., Ichiyanagi, N., Kimura, K. B., Murakami, Y., and Ito, A. (2009) Expression of a soluble isoform of cell adhesion molecule 1 in the brain and its involvement in directional neurite outgrowth. Am. J. Pathol. 174, 2278-2289. doi: 10.2353/ajpath.2009.080743

Heimberger, A. B., Abou-Ghazal, M., Reina-Ortiz, C., Yang, D. S., Sun, W., Qiao, W., et al. (2008). Incidence and prognostic impact of FoxP3+ regulatory $\mathrm{T}$ cells in human gliomas. Clin. Cancer Res. 14, 5166-5172. doi: 10.1158/10780432.CCR-08-0320

Huang, B., Lei, Z., Zhao, J., Gong, W., Liu, J., Chen, Z., et al. (2007). CCL2/CCR2 pathway mediates recruitment of myeloid suppressor cells to cancers. Cancer Lett. 252, 86-92. doi: 10.1016/j.canlet.2006.12.012

Hussain, S. F., Yang, D., Suki, D., Aldape, K., Grimm, E., and Heimberger, A. B. (2006). The role of human glioma-infiltrating microglia/macrophages in mediating antitumor immune responses. Neuro. Oncol. 8, 261-279. doi: 10.1215/15228517-2006-008

Jenkinson, S. R., Williams, N. A., and Morgan, D. J. (2005). The role of intercellular adhesion molecule-1/LFA-1 interactions in the generation of tumor-specific CD8+ T cell responses. J. Immunol. 174, 3401-3407. doi: 10.4049/jimmunol.174.6.3401

Lin, K. C., and Castro, A. C. (1998). Very late antigen 4 (VLA4) antagonists as antiinflammatory agents. Curr. Opin. Chem. Biol. 2, 453-457. doi: 10.1016/S13675931(98)80120-8

Matsui, T., Ishikawa, H., and Bessho, Y. (2015). Cell collectivity regulation within migrating cell cluster during Kupffer's vesicle formation in zebrafish. Front. Cell. Dev. Biol. 3:27. doi: 10.3389/fcell.2015.00027

Nishimura, F., Dusak, J. E., Eguchi, J., Zhu, X., Gambotto, A., Storkus, W. J., et al. (2006). Adoptive transfer of type 1 CTL mediates effective anti-central nervous system tumor response: critical roles of IFN-inducible protein-10. Cancer Res. 66, 4478-4487. doi: 10.1158/0008-5472.CAN-05-3825

Ousman, S. S., and Kubes, P. (2012). Immune surveillance in the central nervous system. Nat. Neurosci. 15, 1096-1101. doi: 10.1038/nn.3161

Palucka, K., and Banchereau, J. (2012). Cancer immunotherapy via dendritic cells. Nat. Rev. Cancer 12, 265-277. doi: 10.1038/nrc3258

Proudfoot, A. E. (2002). Chemokine receptors: multifaceted therapeutic targets. Nat. Rev. Immunol. 2, 106-115. doi: 10.1038/nri722

Rørth, P. (2012). Fellow travellers: emergent properties of collective cell migration. EMBO Rep. 13, 984-991. doi: 10.1038/embor.2012.149

Rothlein, R., Dustin, M. L., Marlin, S. D., and Springer, T. A. (1986). A human intercellular adhesion molecule (ICAM-1) distinct from LFA-1. J. Immunol. $137,1270-1274$. 
Salcedo, R., Ponce, M. L., Young, H. A., Wasserman, K., Ward, J. M., Kleinman, H. K., et al. (2000). Human endothelial cells express CCR2 and respond to MCP-1: direct role of MCP-1 in angiogenesis and tumor progression. Blood 96, 34-40.

Sasaki, K., Zhao, X., Pardee, A. D., Ueda, R., Fujita, M., Sehra, S., et al. (2008). Stat6 signaling suppresses VLA-4 expression by CD8+ T cells and limits their ability to infiltrate tumor lesions in vivo. J. Immunol. 181, 104-108. doi: 10.4049/jimmunol.181.1.104

Sasaki, K., Zhu, X., Vasquez, C., Nishimura, F., Dusak, J. E., Huang, J., et al. (2007). Preferential expression of very late antigen-4 on type 1 CTL cells plays a critical role in trafficking into central nervous system tumors. Cancer Res. 67, 6451-6458. doi: 10.1158/0008-5472.CAN-06-3280

Ueda, R., Kohanbash, G., Sasaki, K., Fujita, M., Zhu, X., Kastenhuber, E. R., et al. (2009). Dicer-regulated microRNAs 222 and 339 promote resistance of cancer cells to cytotoxic T-lymphocytes by down-regulation of ICAM-1. Proc. Natl. Acad. Sci. U.S.A. 106, 10746-10751. doi: 10.1073/pnas.0811817106

Walker, P. R., Calzascia, T., de Tribolet, N., and Dietrich, P. Y. (2003). T-cell immune responses in the brain and their relevance for cerebral malignancies. Brain Res. Brain Res. Rev. 42, 97-122. doi: 10.1016/S0165-0173(03)00141-3

Watabe, K., Ito, A., Koma, Y. I., and Kitamura, Y. (2003). IGSF4: a new intercellular adhesion molecule that is called by three names, TSLC1, SgIGSF and SynCAM, by virtue of its diverse function. Histol. Histopathol. 18, 1321-1329.

Watters, J. J., Schartner, J. M., and Badie, B. (2005). Microglia function in brain tumors. J. Neurosci. Res. 81, 447-455. doi: 10.1002/jnr.20485

Yageta, M., Kuramochi, M., Masuda, M., Fukami, T., Fukuhara, H., Maruyama, T., et al. (2002). Direct association of TSLC1 and DAL-1, two distinct tumor suppressor proteins in lung cancer. Cancer Res. 62, 5129-5133.

Zhu, X., Fujita, M., Snyder, L. A., and Okada, H. (2011). Systemic delivery of neutralizing antibody targeting CCL2 for glioma therapy. J. Neurooncol. 104, 83-92. doi: 10.1007/s11060-010-0473-5

Zhu, X., Nishimura, F., Sasaki, K., Fujita, M., Dusak, J. E., Eguchi, J., et al. (2007). Toll like receptor-3 ligand poly-ICLC promotes the efficacy of peripheral vaccinations with tumor antigen-derived peptide epitopes in murine CNS tumor models. J. Transl. Med. 5:10. doi: 10.1186/14795876-5-10

Zlotnik, A., and Yoshie, O. (2012). The chemokine superfamily revisited. Immunity 36, 705-716. doi: 10.1016/j.immuni.2012.05.008

Zou, W. (2006). Regulatory T cells, tumour immunity and immunotherapy. Nat. Rev. Immunol. 6, 295-307. doi: 10.1038/nri1806

Conflict of Interest Statement: The authors declare that the research was conducted in the absence of any commercial or financial relationships that could be construed as a potential conflict of interest.

Copyright (c) 2015 Fujita, Matsui and Ito. This is an open-access article distributed under the terms of the Creative Commons Attribution License (CC BY). The use, distribution or reproduction in other forums is permitted, provided the original author(s) or licensor are credited and that the original publication in this journal is cited, in accordance with accepted academic practice. No use, distribution or reproduction is permitted which does not comply with these terms. 